\title{
Optical Phonons in Twisted Bilayer Graphene with Gate-Induced Asymmetric Doping
}

\author{
Ting-Fung Chung, ${ }^{* \dagger, \ddagger}$ Rui He, ${ }^{*} \S$ Tai-Lung Wu, ${ }^{\dagger, \ddagger}$ and Yong P. Chen ${ }^{\dagger,+, \|}$
}

${ }^{\dagger}$ Department of Physics and Astronomy and ${ }^{\ddagger}$ Birck Nanotechnology Center, Purdue University, West Lafayette, Indiana 47907, United States

${ }^{\S}$ Department of Physics, University of Northern Iowa, Cedar Falls, Iowa 50614, United States

"School of Electrical and Computer Engineering, Purdue University, West Lafayette, Indiana 47907, United States

\section{Supporting Information}

ABSTRACT: Twisted bilayer graphene (tBLG) devices with ion gel gate dielectrics are studied using Raman spectroscopy in the twist angle regime where a resonantly enhanced $G$ band can be observed. We observe prominent splitting and intensity quenching on the $G$ Raman band when the carrier density is tuned away from charge neutrality. This $G$ peak splitting is attributed to asymmetric charge doping in the two graphene layers, which reveals individual phonon self-energy renormalization of the two weakly coupled layers of graphene. We estimate the effective interlayer capacitance at low doping density of tBLG using an interlayer screening model. The anomalous intensity quenching of both $G$ peaks is ascribed to the

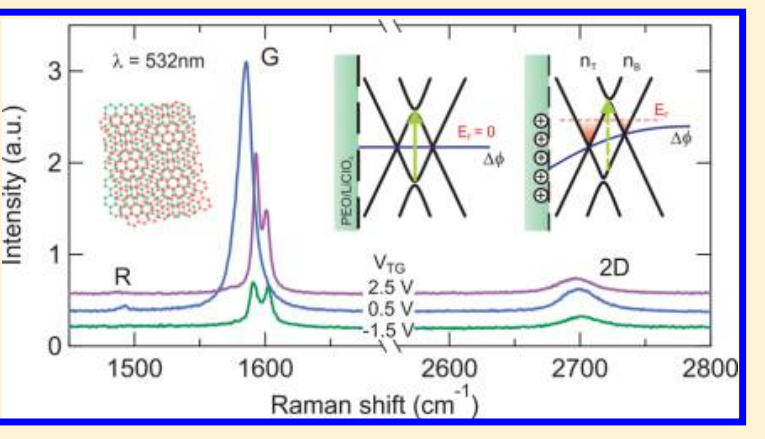
suppression of resonant interband transitions between the two saddle points (van Hove singularities) that are displaced in the momentum space by gate-tuning. In addition, we observe a softening (hardening) of the R Raman band, a superlattice-induced phonon mode in tBLG, in electron (hole) doping. Our results demonstrate that gate modulation can be used to control the optoelectronic and vibrational properties in tBLG devices.

KEYWORDS: Twisted bilayer graphene, gating, Raman, interlayer screening

$\mathrm{R}$ ecently there has been growing interest in two-dimensional (2D) van der Waals (vdW) materials and structures in which interlayer interaction can significantly affect these systems' properties and functionalities. ${ }^{1-9}$ Twisted bilayer graphene (tBLG) in which the two graphene layers are stacked with a twist angle $(\theta)$ and coupled by vdW force has been demonstrated to show new physical (electronic, vibrational, and optical) properties through changed interlayer interaction at different twist angles. ${ }^{10-15}$ Angle-resolved photoemission spectroscopy measurement has shown that tBLG exhibits weak interlayer coupling as revealed by the presence of van Hove singularities (VHSs) in the density of states at the overlap (saddle point) of two single layer graphene (SLG) Dirac cones. ${ }^{13}$ Furthermore, low-energy, $\theta$-dependent VHSs and superlattice Dirac cones have been observed by scanning tunneling microscopy and spectroscopy. ${ }^{16-18}$ Optical spectroscopy has been exploited to study the optical and vibrational properties associated with the low-energy VHSs of tBLG. ${ }^{19-27}$ These studies have demonstrated that $\mathrm{tBLG}$ is a prototype system to explore the influence of interlayer interaction in $2 \mathrm{D}$ layered materials.

Raman spectroscopy is a sensitive probe of the unique electronic and phonon band structures of tBLG through resonance enhancement and superlattice induced Raman processes. Raman intensities from $G$ and double-resonant (DR) $\mathrm{ZO}_{\mathrm{L}}^{\prime}$ (fundamental layer-breathing vibration) bands display large resonance enhancements when the excitation photon energy equals to the inter-VHS energy ( $E_{\mathrm{VHS}}$; the energy difference between the saddle points in the conduction and valence bands). ${ }^{19-22,27}$ The twist angle at which $E_{\mathrm{VHS}}$ equals the excitation photon energy is called the critical angle $\theta_{c}$. For $532 \mathrm{~nm}$ laser excitation, $\theta_{c}$ is $\sim 12.5^{\circ}$, and it becomes $\sim 10.5^{\circ}$ for $633 \mathrm{~nm}$ laser excitation. ${ }^{22}$ Several new Raman bands, such as $\mathrm{R}, \mathrm{R}^{\prime}$ and $\mathrm{ZO}_{\mathrm{H}}^{\prime}$, are activated by superlatticeinduced wavevector. ${ }^{25-27,29,30}$ These characteristic Raman features related to the low-energy VHSs and superlattice atomic structure have been observed only in $\mathrm{tBLG}^{19-31}$ but not in SLG or Bernal-stacked bilayer graphene (AB-BLG).

In this Letter, we report the observation of gate-induced $G$ Raman band splitting and intensity quenching in tBLG with twist angle close to $\theta_{\mathcal{c}}$ at which the ungated sample exhibits a large $G$ band resonance enhancement. By creating an asymmetric doping in the two layers via electrochemical gating, the Raman spectra of tBLG evolve in ways that differ greatly from those observed for SLG and AB-BLG. The observed G band splitting shows no optical phonon mixing, suggesting the absence of the infrared (IR)-active $\mathrm{E}_{\mathrm{u}}$ mode that is present in

Received: November 10, 2014

Revised: December 29, 2014

Published: January 26, 2015 
gated AB-BLG devices. ${ }^{32,33}$ In our studies, we are able to estimate the Fermi energy $E_{\mathrm{F}}$ and carrier density in each graphene layer from the split $G$ Raman bands which show individual phonon self-energy renormalizations (with different charge carrier densities in the two layers). An interlayer screening model is employed to explain the G band splitting of the tBLG with doping asymmetry. An effective interlayer capacitance of $\sim 4.6 \mu \mathrm{Fcm}^{-2}$ is estimated from the interlayer potential between two graphene layers. The unusual G Raman intensity quenching away from the CNP is attributed to the reduction in the joint density of states (JDOS) associated with interband transition near VHSs in which the saddle points are displaced in both energy and momentum by the interlayer potential. ${ }^{34}$ In addition, the dependence of the $\mathrm{R}$ Raman band on the gate voltage (carrier density) was observed for the first time. Its phonon self-energy renormalization could have contribution from both electron-phonon and electronelectron interactions, similar to what occurs in the $2 \mathrm{D}$ Raman band.

Our graphene samples were grown on $\mathrm{Cu}$ foils by chemical vapor deposition and transferred onto a heavily p-doped $\mathrm{Si}$ substrate (coated with $\sim 300 \mathrm{~nm} \mathrm{SiO}_{2}$ ). ${ }^{27}$ Field effect devices were fabricated for Raman studies and electrical characterizations. Figure 1a shows an optical image of the fabricated graphene device, which consists of SLG (upper) and tBLG (lower) regions (delimited by the dashed white line). A color contrast between tBLG (darker) and SLG can be seen. Raman studies from both the SLG and tBLG regions of the device were performed. From the R, G, and 2D Raman characteristics, we determined that the twist angle $\theta$ of the tBLG is about $13^{\circ},{ }^{21,22,29}$ close to the critical angle $\theta_{\mathrm{c}}\left(12.5^{\circ}\right.$ for the excitation photon energy of $2.33 \mathrm{eV}$ of a $532 \mathrm{~nm}$ laser). The Raman G band intensity is strongly enhanced at this twist angle because the energy between the saddle points (VHSs) in the conduction and valence bands is resonant with the photon energy of the incident laser beam. ${ }^{21,22}$ Figure $1 \mathrm{~b}$ shows a schematic drawing of our device setup for electrochemical gating. A voltage $\left(V_{\mathrm{TG}}\right)$ applied to the side electrode is used to gate the graphene via the ion gel dielectrics $\left(\mathrm{PEO} / \mathrm{LiClO}_{4}\right)$ that acts as a top gate. Details of the sample growth and experimental procedures can be found in the Supporting Information.

Figure 1c displays three representative Raman spectra from tBLG at different $V_{\mathrm{TG}}$. Spectra from the SLG are included for comparison. All spectra are normalized to the $520 \mathrm{~cm}^{-1}$ Si peak. At $V_{\mathrm{TG}} \sim 0.5 \mathrm{~V}$, the $\mathrm{R}, \mathrm{G}$, and $2 \mathrm{D}$ bands from the $\mathrm{tBLG}$ are at about 1492, 1584, and $2699 \mathrm{~cm}^{-1}$, respectively. We estimate the charge neutrality point $(\mathrm{CNP})$ voltage $V_{\mathrm{D}} \sim 0.5 \mathrm{~V}$ based on the approximate symmetry of the spectra evolution with respect to electron (n-) and hole (p-) doping (also see Figures 2 and 3a) away from this voltage. The nonzero $V_{\mathrm{D}}$ is ascribed to unintentional extrinsic doping from the $S i$ substrate and the ion gel electrolyte. ${ }^{32,35}$ The positive and negative signs of the $\left(V_{\mathrm{TG}}\right.$ $\left.-V_{\mathrm{D}}\right)$ correspond to $\mathrm{n}$ - and $\mathrm{p}$-doping in graphene, respectively. Doping dependence of the G and 2D bands from the SLG in our device is in good agreement with previous reports. ${ }^{36-38}$ The $V_{\mathrm{D}}$ of the SLG is determined to be $\sim 0.6 \mathrm{~V}$, slightly higher than that of the tBLG. This is also consistent with the $V_{\mathrm{D}}$ values determined by electrical transport measurement (Figure S5 in the Supporting Information). As shown in Figure 1c, the G Raman band from tBLG exhibits strong resonance enhancement (intensity $\sim 40$ times larger compared to that of SLG at the CNP).

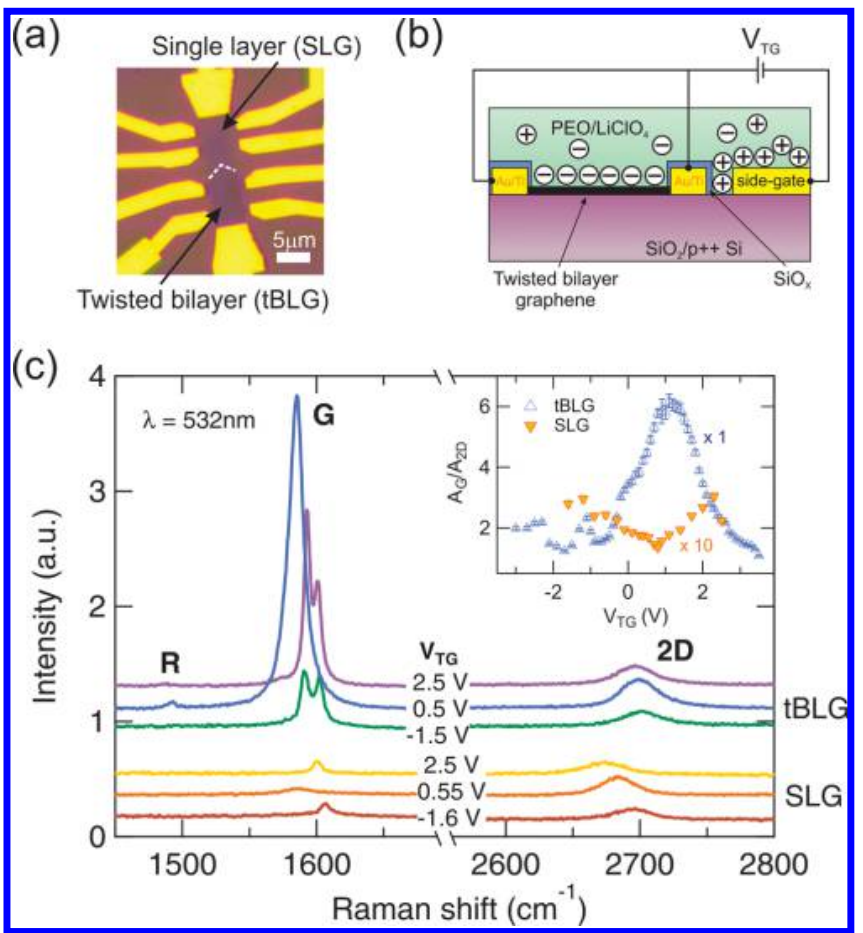

Figure 1. (a) An optical image of an electrochemically top-gated single layer (SLG) and twisted bilayer graphene (tBLG) device before application of ion gel electrolyte. The sample consists of a SLG (upper part) and a tBLG (lower part). The two parts show different optical contrast. The boundary between the SLG and tBLG is delimited by a dashed white line. (b) Schematic of device configuration (for the case with negative electrolyte top-gate voltage $V_{\mathrm{TG}}$ ). (c) Comparison of Raman spectra of the SLG and tBLG at several different gate voltages $V_{\mathrm{TG}}$. Spectra are normalized to the height intensity of the $520 \mathrm{~cm}^{-1} \mathrm{Si}$ peak and are shifted vertically for clarity. All data were taken at room temperature using a $532 \mathrm{~nm}$ laser excitation. The charge neutrality point $(\mathrm{CNP})$ voltage $\left(V_{\mathrm{D}}\right)$ of the SLG and tBLG is $\sim 0.6$ and $\sim 0.5 \mathrm{~V}$, respectively, as estimated from the minimum of $G$ band frequency (Figure 3a). The sample is electron (n)-doped for $V_{\mathrm{TG}}>V_{\mathrm{D}}$ and is hole (p)-doped otherwise. The vertical scale is the same before and after the break on the horizontal axis. The upper right inset shows the ratios of the integrated intensities of the $\mathrm{G}$ and $2 \mathrm{D}$ peaks $\left(A_{\mathrm{G}} / A_{2 \mathrm{D}}\right)$ as a function of $V_{\mathrm{TG}}$ from both the SLG and tBLG. The data of the SLG in the inset is multiplied by a factor of 10 for clarity.

Figures $1 \mathrm{c}$ and 2 show that the G band of the tBLG not only blueshifts but also splits into two peaks when $V_{\mathrm{TG}}$ is away from $V_{\mathrm{D}}$. Near the CNP, the spectra are described by a single Lorentzian line shape with a full width at half-maximum (fwhm) of $\sim 15 \mathrm{~cm}^{-1}$, comparable to that of charge neutral SLG. In SLG the G band only shows a blueshift without splitting when $V_{\mathrm{TG}}$ is tuned away from the CNP (see Figure 1c and Supporting Information Figure S1). This frequency upshift is well-studied and explained by phonon self-energy renormalization due to electron-phonon coupling (EPC). ${ }^{36,38}$ Although a uniaxial strain may induce a splitting of the $G$ band for the SLG, ${ }^{39,40}$ such a G band splitting is not observed (see Figure 1c and Supporting Information Figure S1) in the SLG region in our device, indicating that strain is negligible in our fabricated devices. This further indicates that the observed G splitting in the tBLG (lower region in the same device, see Figure 1a) is unlikely to be associated with strain. ${ }^{41}$

In gated AB-BLG device, it has been shown that the $\mathrm{G}$ band splits due to optical phonon mixing (symmetric $E_{g}$ and asymmetric $E_{u}$ ) when the $A B$ sublattice symmetry is broken by 


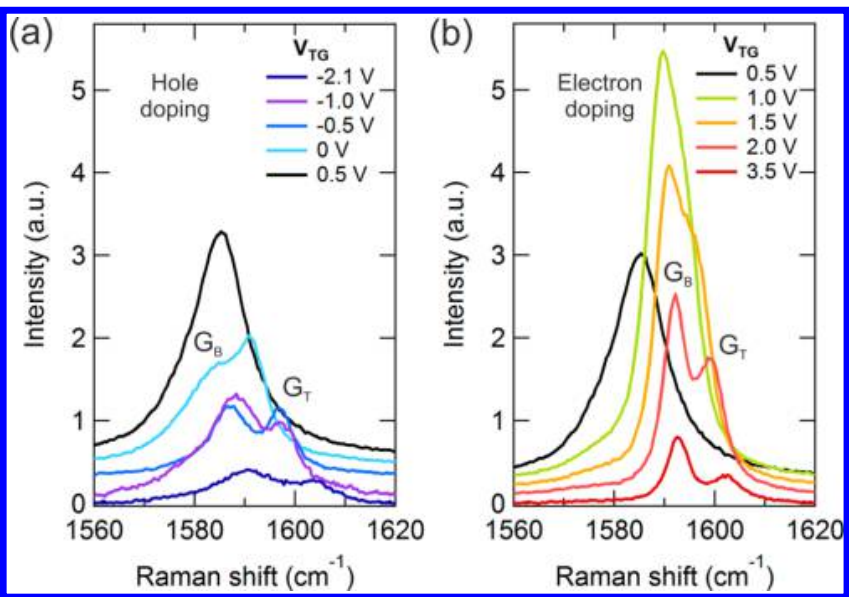

Figure 2. (a) Evolution of the normalized Raman spectrum in the region of the $\mathrm{G}$ band in the tBLG as a function of $V_{\mathrm{TG}}$ in p-doped regime. (b) Same as in (a) for n-doped regime. The spectra at $V_{\mathrm{D}} \sim$ $0.5 \mathrm{~V}$ are plotted with black line. The doublet $\mathrm{G}$ bands are denoted as $G_{B}$ and $G_{T}$ peaks in which the subscripts $B$ and $T$ represent the bottom and top graphene layer, respectively, in the tBLG. Spectra are shifted vertically for clarity.

application of an out-of-plane electric field, where the oddparity $\mathrm{E}_{\mathrm{u}}$ mode becomes active in Raman scattering. ${ }^{32,33}$ In $\mathrm{tBLG}$, the $\mathrm{AB}$ sublattice symmetry is naturally broken because of the relative rotation between the two layers regardless of the charge doping. However, the $\mathrm{E}_{\mathrm{u}}$ mode has not been observed in Raman studies of tBLG under zero gate voltage, ${ }^{21,22,32,35,42}$ suggesting this $E_{u}$ mode remains Raman-inactive or silent in tBLG. Araujo et al. and Kalbac et al. studied the Raman features of twisted bilayer ${ }^{12} \mathrm{C} /{ }^{13} \mathrm{C}$ graphene with large twist angle using electrochemical doping method. ${ }^{35,42}$ No obvious signature of the $E_{u}$ mode has been observed. In addition, the doublet $G$ lines observed in our doped tBLG sample are different from those reported on AB-BLG (optical phonon mixing) in which the two G Raman peaks give opposite frequency shift, while simultaneously a reversal of resonance intensities occurs with increasing doping density. ${ }^{32,33}$ However, in our doped tBLG sample we observe a concurrent upshift of the doublet $G$ lines (Figures 1c and 2) and reduction of their intensities without crossing when increasing carrier density. Therefore, the $G$ band splitting in our tBLG device is not caused by such optical phonon mixing. Instead, we attribute the splitting to the gateinduced asymmetric doping in the two layers of the tBLG such that each layer gives $G$ band, to be discussed in more details later.

Significant quenching of the resonantly enhanced G Raman intensity with increasing doping level is also observed in the tBLG. Ratios of the integrated intensities of the $G$ and $2 D$ bands $\left(A_{\mathrm{G}} / A_{2 \mathrm{D}}\right)$ in the tBLG and SLG as functions of $V_{\mathrm{TG}}$ are shown in the inset of Figure 1 (c). The $A_{\mathrm{G}} / A_{2 \mathrm{D}}$ of the tBLG is at its maximum at $\sim 1 \mathrm{~V}$, which is higher than the estimated $V_{\mathrm{D}}$ of $\sim 0.5 \mathrm{~V}$, and then $A_{\mathrm{G}} / A_{2 \mathrm{D}}$ drastically declines by a factor of up to 6 while the sample is heavily doped. In contrast, this $A_{\mathrm{G}} / A_{2 \mathrm{D}}$ ratio in the SLG is at its minimum very close to the CNP $\left(V_{\mathrm{D}} \sim\right.$ $0.6 \mathrm{~V}),^{36}$ and it is enhanced by a factor of $\sim 3$ when the sample is heavily doped. The increase of $A_{\mathrm{G}} / A_{2 \mathrm{D}}$ in SLG has been ascribed to the reduction of $A_{2 \mathrm{D}}$ (Supporting Information Figure S2) due to an increase of scattering between photoexcited carriers as the doping level increases. ${ }^{43,44}$ This doping dependence of the $2 \mathrm{D}$ intensity also occurs in the tBLG
(Figure 1c). However, the G band intensity may decrease even faster than the $2 \mathrm{D}$ intensity in tBLG as the doping level increases. Among all the Raman bands seen in the $\mathrm{tBLG}$ device (Figure 1c), the $\mathrm{G}$ band intensity shows the strongest resonance at $\sim 1 \mathrm{~V}$ (see Figure $2 \mathrm{~b}, \sim 0.5 \mathrm{~V}$ above the CNP), which suggests that the energy separation between the VHSs is not well overlapped with the incident photon energy at CNP (slightly off-resonance). This energy difference could be attributed to disorder (unintentional doping or strain caused by wrinkles) and the fact that the twist angle is slightly different from the critical angle. The $G$ band intensity is subject to the greatest suppression when the sample is further doped, implying strong influence of the doping on the resonance condition. Although the Fermi level in our experiment $\left(\left|E_{\mathrm{F}}\right|\right.$ can be tuned $\sim 0.5 \mathrm{eV}$ away from $\mathrm{CNP}$ ) cannot reach the $E_{\mathrm{VHS}}$ $(\sim \pm 1 \mathrm{eV}$ from CNP) of the tBLG, the resonance condition can still be modulated by gating. The strong $G$ Raman band intensity quenching in the tBLG is attributed to off-resonance or the reduced JDOS associated with the VHSs due to gating and will be discussed later in this paper.

Figure $3 a-c$ shows the peak frequencies $\omega_{G}$, fwhms $\Gamma_{G}$, and integrated intensities $A_{\mathrm{G}}$ of the doublet $\mathrm{G}$ peaks $\left(\mathrm{G}_{\mathrm{T}}\right.$ and $\mathrm{G}_{\mathrm{B}}$, the subscripts $\mathrm{T}$ and $\mathrm{B}$ represent top and bottom layers, respectively) as a function of $V_{\mathrm{TG}}$. It is reasonable to assign the layer which has more prominent changes in the $G$ features as the top layer since this layer is in direct contact with the top ion gate electrolyte and is more strongly influenced by the gating. All parameters are extracted from simple fits of the bands with two Lorentzian peaks. A single Lorentzian function is used to fit the unsplit $\mathrm{G}$ peak in the vicinity of the CNP. These data points are shown by solid blue squares in Figure $3 a-c$. With increasing carrier density, the frequencies of the two $G$ peaks blueshift at different rates and their intensities decrease simultaneously, indicating the off-resonance condition when $V_{\mathrm{TG}}$ is away from the CNP. These features are very different from the gate dependence of the $G$ doublet peaks in AB-BLG in which the two $G$ peaks repel each other in energy, and a reversal of their intensities takes place and crosses at around $200 \mathrm{meV}$ with respect to the CNP. In our case of the doped tBLG, the two G peaks appear to be uncoupled to each other in frequency and fwhm, and show no crossing in their intensities. Unlike $A B-$ BLG, the observed doping dependence of both $G_{T}$ and $G_{B}$ peaks in the tBLG agree quite well with those observed in SLG (Supporting Information Figure S1) in which the frequency (fwhm) blueshifts (narrows) with increasing charge density. The $\Gamma_{\mathrm{G}}$ changes by $\Delta \Gamma_{\mathrm{G}} \sim 8.7 \pm 0.5 \mathrm{~cm}^{-1}$ for both the $\mathrm{G}_{\mathrm{T}}$ and $\mathrm{G}_{\mathrm{B}}$ peaks as $V_{\mathrm{TG}}$ is tuned away from the CNP (Figure $3 \mathrm{~b}$ and inset of Figure $3 \mathrm{~d}$ ). Following the similar method used to estimate the EPC strength in SLG from $\Delta \Gamma_{\mathrm{G}}{ }^{38,45}$ we estimate that the EPC strength of each graphene layer in the tBLG is $14.3 \pm 0.4 \mathrm{eV} / \AA$, comparable to that of SLG $\left(\Delta \Gamma_{\mathrm{G}} \sim 8.5 \mathrm{~cm}^{-1}\right.$ and EPC strength of $\sim 14.1 \mathrm{eV} / \AA){ }^{38}$ This finding reveals that the interlayer interaction between the two graphene layers in the tBLG is sufficiently weak and has negligible effect on the EPC of the intralayer G phonons for each layer, which behaves similarly to a SLG.

We have calculated the carrier densities (doping) of each layer (top/bottom) from the corresponding $G$ Raman peak $\left(G_{T} / G_{B}\right)$ frequencies, assuming similar dependence of the $G$ peak frequency as that found for a SLG. It has been experimentally shown that the $G$ peak blueshifts linearly with $E_{\mathrm{F}}$ in SLG $\left.\left(\Delta \omega_{\mathrm{G}} \propto E_{\mathrm{F}}\right)\right)^{38,46,47}$ This feature is confirmed in our SLG (Figure S1, eqs S3 and S4 in Supporting Information), 


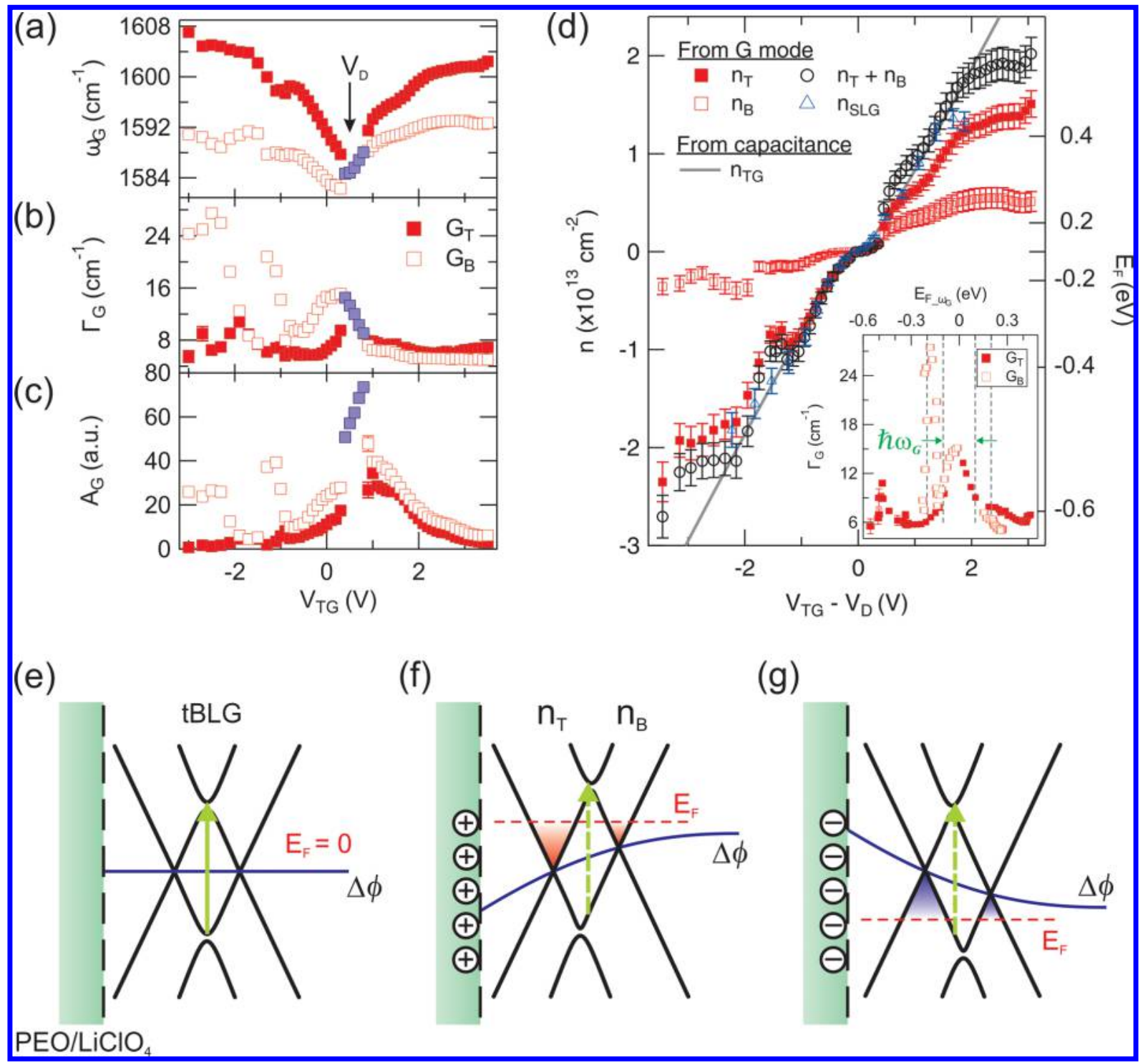

Figure 3. $(\mathrm{a}-\mathrm{c})$ Evolution of the frequency $\left(\omega_{\mathrm{G}}\right)$, fwhm $\left(\Gamma_{\mathrm{G}}\right)$, and integrated intensity $\left(A_{\mathrm{G}}\right)$ of the $\mathrm{G}_{\mathrm{B}}$ and $\mathrm{G}_{\mathrm{T}}$ peaks as a function of $V_{\mathrm{TG}}$. The blue solid squares correspond to $G$ peaks that show single Lorentzian line shape (no splitting). (d) Carrier densities (doping) calculated either from Raman G peak position assuming SLG behavior or from gate capacitance. The total density of the tBLG $\left(n_{\mathrm{T}}+n_{\mathrm{B}}\right)$ is in good agreement with that of SLG $\left(n_{\mathrm{SLG}}\right)$. The induced carrier density in SLG estimated from the gate and quantum capacitances ( $n_{\mathrm{TG}}$, Supporting Information eq S2) is plotted for comparison. The inset shows the evolution of the $\mathrm{G}_{\mathrm{B}}$ and $\mathrm{G}_{\mathrm{T}}$ fwhms as a function of the effective Fermi energy $E_{\mathrm{F}_{-} \omega_{\mathrm{G}}}$ of each individual layer. (e) Schematic energy band diagram of tBLG when it is charge neutral. Significant interband transitions (solid green arrow) are indicated that give rise to strong resonance enhancement on the $G$ band. (f) Same as in (e) for $n$-doped situation assuming the two layers are in equilibrium (same chemical potential indicated by $E_{\mathrm{F}}$ ). Electric-field screening results in an interlayer potential offset $(\Delta \phi)$ between the layers, resulting in the higher charge carrier density in the top layer $\left(\left|n_{\mathrm{T}}\right|>\left|n_{\mathrm{B}}\right|\right)$. The dashed green arrow shows interband direct transitions that are diminished due to the shift of the two Dirac cones, leading to the intensity quenching of the $G$ bands. (g) Same as in (f) for p-doped situation.

yielding a linear relation $\left|E_{\mathrm{F}}\right| \times 40=\omega_{\mathrm{G}}-1583.8\left(\left|E_{\mathrm{F}}\right| \times 45=\right.$ $\left.\omega_{\mathrm{G}}-1583.8\right)$ for $\mathrm{n}-(\mathrm{p}-)$ doped SLG, where $E_{\mathrm{F}}$ and $\omega_{\mathrm{G}}$ are in units of $\mathrm{eV}$ and $\mathrm{cm}^{-1}$, respectively, in good agreement with prior studies. $^{38,46,47}$ Taking into account of different minimum G Raman peak frequencies $\left(\sim 2 \mathrm{~cm}^{-1}\right)$ in tBLG $\left(\sim 1583 \mathrm{~cm}^{-1}\right)$ and SLG $\left(\sim 1585 \mathrm{~cm}^{-1}\right)$, we extract the $E_{\mathrm{F}}$ and carrier concentration $n=\left(E_{\mathrm{F}} / \hbar v_{\mathrm{F}}\right)^{2} / \pi$ (from linear E-k dispersion), in the top $\left(n_{\mathrm{T}}\right)$ and bottom $\left(n_{\mathrm{B}}\right)$ layers of the tBLG (Figure $3 \mathrm{~d}$ ) using modified relations in the form of $\left|E_{\mathrm{F}}\left(n_{\mathrm{T}}\right)\right| \times 42=\omega_{\mathrm{G}}\left(n_{\mathrm{T}}\right)$ - 1582 and $\left|E_{\mathrm{F}}\left(n_{\mathrm{B}}\right)\right| \times 42=\omega_{\mathrm{G}}\left(n_{\mathrm{B}}\right)-1582$, respectively. The error bars (Figure $3 \mathrm{~d}$ ) include the uncertainties of the two numerical values used (42 and 1582). The total carrier concentration $n_{\text {total }}=n_{\mathrm{T}}+n_{\mathrm{B}}$ of the tBLG system is shown as empty black circles in Figure 3d. The charge density in the SLG (denoted as $n_{\mathrm{SLG}}$ ) is also calculated from its G Raman frequency (Supporting Information eqs S3 and S4) and shown as empty blue triangles. Finally, the effective charge density (denoted as $n_{\mathrm{TG}}$ ) induced by the ion gel gating on SLG is calculated by $e\left(V_{\mathrm{TG}}-V_{\mathrm{D}}\right)=n_{\mathrm{TG}} e^{2} / C_{\mathrm{TG}}+\hbar v_{\mathrm{F}}\left(n_{\mathrm{TG}} \pi\right)^{1 / 2}$ (see eq $\mathrm{S} 2$ in Supporting Information) and shown as a solid gray line in Figure $3 \mathrm{~d}^{36}$ where $\mathrm{C}_{\mathrm{TG}} \approx 2 \mu \mathrm{Fcm}^{-2}$ is the capacitance of the electrolyte and agrees with prior reports, ${ }^{36} V_{\mathrm{TG}}-V_{\mathrm{D}}$ is the applied voltage relative to $\mathrm{CNP}$, and $e$ is the electron charge. The first and second terms are ascribed to geometric and quantum capacitances, respectively. At low doping $\left(|\Delta V|=\mid V_{\mathrm{TG}}\right.$ $-V_{\mathrm{D}} \mid$ up to $\sim 2 \mathrm{~V}$; equivalent to $|n|$ up to $\sim 1.8 \times 10^{13} \mathrm{~cm}^{-2}$ ), 
$n_{\text {total }}=n_{\mathrm{T}}+n_{\mathrm{B}}$ for the tBLG calculated from the $\mathrm{G}_{\mathrm{T}} / \mathrm{G}_{\mathrm{B}}$ Raman peaks agrees well with $n_{\mathrm{TG}}$ and $n_{\mathrm{SLG}}$. When $|\Delta V|>2 \mathrm{~V}, n_{\text {total }}=$ $n_{\mathrm{T}}+n_{\mathrm{B}}$ deviates notably from both $n_{\mathrm{SLG}}$ and $n_{\mathrm{TG}}$, possibly due to a reduced gating efficiency of electrolyte at relatively high gate voltages. We also notice more electron-hole asymmetry in $n_{\text {total }}$ at such large gate biases. The consistency between the doping density extracted from G Raman peaks (based on the assumption that each layer behaves as SLG) of the tBLG and those expected from the capacitance and measured from the SLG confirms that the coupling between the two layers in our tBLG system is sufficiently weak such that each layer retains its SLG-like low energy electronic structure (Dirac band dispersion) and phonon self-energy renormalization (dependence of $\mathrm{G}$ peak frequency on $E_{\mathrm{F}}$ ). On the other hand, we point out that the coupling between the two layers still exists, giving rise to the VHSs at higher energies due to the coupling between Dirac cones from the two graphene layers, as manifested by the resonantly enhanced $\mathrm{G}$ band observed near CNP.

The difference in the gate-dependence of the $G_{T}$ and $G_{B}$ peaks also reflects the difference in the phonon renormalization magnitudes due to different carrier densities in the two graphene layers. As shown in Figure 3d, the carrier density in the bottom layer $\left(n_{\mathrm{B}}\right)$ becomes almost constant around $\pm 0.4 \times$ $10^{13} \mathrm{~cm}^{-2}$ when $|\Delta V|>2 \mathrm{~V}$, and additional doping mainly contributes to the top layer. This leads to continued increase in the peak frequency of $\mathrm{G}_{\mathrm{T}}$ but saturation of the peak frequency of $\mathrm{G}_{\mathrm{B}}$ upon further increasing of $|\Delta V|$ (Figure 3a). The inset of Figure $3 \mathrm{~d}$ displays the $\Gamma_{\mathrm{G}}$ of the doublet $\mathrm{G}$ peaks as a function of the Fermi energy $\left(E_{\mathrm{F} \omega_{\mathrm{G}}}\right.$ estimated from the $\mathrm{G}_{\mathrm{T}}$ and $\mathrm{G}_{\mathrm{B}}$ phonon frequencies) in each layer. We note the similarity of the line shape between the top and bottom layers within $E_{\mathrm{F} \_} \omega_{\mathrm{G}}= \pm$ $0.2 \mathrm{eV}$. Furthermore, the widths of the two $\Gamma_{\mathrm{G}}$ vs $E_{\mathrm{F}-\omega_{\mathrm{G}}}$ peaks are close to the phonon energy $\hbar \omega_{\mathrm{G}}(\sim 200 \mathrm{meV})$, indicating Landau damping of the $\mathrm{G}$ phonons which decay into electronhole pairs. ${ }^{38}$

Figure $3 \mathrm{e}-\mathrm{g}$ schematically illustrates an interlayer screening model that we propose to describe the G band splitting and Raman intensity quenching observed in tBLG. The charge distribution over the top $\left(n_{\mathrm{T}}\right)$ and bottom $\left(n_{\mathrm{B}}\right)$ layers depends on the electrostatic interaction between layers and bandfilling. ${ }^{48,49}$ Both the top and bottom graphene layers are in direct contact with each other and with the metal electrodes. Therefore, the $E_{\mathrm{F}}$ 's of the two layers are assumed to be aligned when the system is in equilibrium. In the undoped tBLG (ideal flat band condition), there is no potential difference $(\Delta \phi)$ between the two layers, and the $E_{\mathrm{F}}$ is at the CNP. In this case, the conduction and valence bands near the saddle points (VHSs) of tBLG are aligned and parallel to each other, maximizing the JDOS for resonant interband transitions (green arrows) between the VHSs, ${ }^{10,22,34}$ and a very strong enhancement of the G Raman band appears.

An accumulation of positive ions in the electrolyte results in $\mathrm{n}$-doped tBLG (Figure 3f). The doping is more efficient in the top layer because the electrolyte ions are closer to the top layer than to the bottom layer (in contact with the Si substrate). The two layers of the $\mathrm{tBLG}$ share the same aligned $E_{\mathrm{F}}$ (dashed red line). However, their CNPs are lifted by an interlayer potential $(\Delta \phi)$. The top and bottom layers of the tBLG feel different electric fields $E_{\mathrm{T}}=\left(n_{\mathrm{T}}+n_{\mathrm{B}}\right) e / \varepsilon_{\mathrm{PE}} \varepsilon_{0}$ and $E_{\mathrm{B}}=n_{\mathrm{B}} e / \varepsilon_{\mathrm{G}} \varepsilon_{0}$, where $\varepsilon_{\mathrm{PE}}$ and $\varepsilon_{\mathrm{G}}$ are the relative dielectric constants of the electrolyte and graphene, respectively, $\varepsilon_{0}$ is the vacuum permittivity. The difference in the electric field $\left(E_{\mathrm{T}}-E_{\mathrm{B}}>0\right)$ is attributed to electronic screening by the charge carriers of the top layer and different $\varepsilon_{\mathrm{PE}}$ and $\varepsilon_{\mathrm{G}}$. Indeed, the electronic screening plays a crucial role in creating charge density asymmetry in graphene layers, and the strength of the screening depends on the doping level as studied by Kuroda et al. ${ }^{49}$ The screening length corresponding to our doping level of $10^{13} \mathrm{~cm}^{-2}$ is only a fraction of the graphene interlayer spacing $\left(d_{0} \approx 0.34 \mathrm{~nm}\right) .{ }^{49}$ The strong resonance enhancement on the G Raman band in the flat band case originates from the resonant interband transitions between the saddle points in the absence of the interlayer potential. ${ }^{21,22}$ In the presence of the interlayer potential, the saddle points are oppositely displaced in momentum space and the electronic band structure is altered (Figure 3f). ${ }^{10,34,50}$ Direct interband transitions connecting the two saddle points (VHSs) become forbidden (in this sense the energy separation between the saddle points become "indirect", as demonstrated by the dashed green arrow, in analogy with an indirect bandgap in semiconductors). Therefore, the JDOS of the system for the interband transition (between VHSs) and the resonant $\mathrm{G}$ band enhancement are suppressed. Note that this mechanism is different from the modification of JDOS (optical absorption) caused by the many-body effects (electron-hole and electron-electron interactions) in doped SLG. ${ }^{51}$ Similar explanation is applicable to the p-doped tBLG (Figure 3g).

We can quantitatively describe the $n_{\mathrm{T}}$ and $n_{\mathrm{B}}$ dependence on $V_{\text {TG }}$ using the band diagrams shown in Figure 3f,g. An applied $\left(V_{\mathrm{TG}}-V_{\mathrm{D}}\right)$ is the sum of potential drop across the Debye length of the electrolyte ${ }^{36}$ (due to electrostatic capacitance) and the Fermi energy (with respect to CNP) of the top layer (due to quantum capacitance): $e\left(V_{\mathrm{TG}}-V_{\mathrm{D}}\right)=e^{2}\left(n_{\mathrm{T}}+n_{\mathrm{B}}\right) / C_{\mathrm{TG}}+$ $E_{\mathrm{F}}\left(n_{\mathrm{T}}\right)$, here $E_{\mathrm{F}}\left(n_{\mathrm{T}}\right)$ is positive (negative) for electron (hole) carriers. Similarly, the Fermi energy of the top layer can be written as the sum of the Fermi energy (with respect to CNP) of the bottom layer and the interlayer potential: $E_{\mathrm{F}}\left(n_{\mathrm{T}}\right)=$ $E_{\mathrm{F}}\left(n_{\mathrm{B}}\right)+\Delta \phi$. If we treat the two layers of $\mathrm{tBLG}$ as a simple parallel-plate capacitor, the interlayer potential is $\Delta \phi=E_{\mathrm{F}}\left(n_{\mathrm{T}}\right)$ - $E_{\mathrm{F}}\left(n_{\mathrm{B}}\right)=e^{2} n_{\mathrm{B}} / C_{\mathrm{tBLG}}$ (Supporting Information Figure S3), where $C_{\mathrm{tBLG}}$ is the effective interlayer static capacitance per unit area of graphene. Note that $\Delta \phi$ is positive (negative) in $\mathrm{n}-(\mathrm{p}-)$ doped tBLG. From the above analysis, we determine the effective interlayer static capacitance $C_{\mathrm{tBLG}} \sim 4.6 \mu \mathrm{Fcm}^{-2}$ from the slopes (linear blue lines for both carriers) in Supporting Information Figure $\mathrm{S} 3$, close to $5.2-7.8 \mu \mathrm{Fcm}^{-2}$ estimated from $C_{\mathrm{tBLG}}=\varepsilon_{\mathrm{G}} \varepsilon_{0} / d_{0}$. Here, the relative dielectric constant of BLG is $\varepsilon_{\mathrm{G}}=2-3{ }^{52}$ and $d_{0}=0.34 \mathrm{~nm}$ is used in this estimation.

We note that the CNP of the top and bottom layers in the tBLG are slightly different (by $\sim 0.2 \mathrm{~V}$ ) (see Figure $3 \mathrm{a}$ ). This asymmetry may be attributed to unintentional doping by the substrate and nonuniform doping by the polymer electrolyte. The bottom layer is in direct contact with thus subject to a stronger influence from the substrate. It has been shown that charged impurities can be trapped at the tBLG/substrate interface in the graphene transfer process. These impurities may cause the two graphene layers to respond differently during gating. ${ }^{53}$ In addition, the polymer electrolyte may dope the two graphene layers differently at $V_{\mathrm{TG}}=0$ (the top layer is doped with more carriers on the order of $\sim 10^{12} \mathrm{~cm}^{-2}$ because it is in contact with the electrolyte). ${ }^{35}$ We also note that there are discontinuities in the gate dependent $G$ frequencies, fwhms, and integrated intensities when $V_{\mathrm{TG}} \sim-1.5 \mathrm{~V}\left(E_{\mathrm{F}} \sim-0.4 \mathrm{eV}\right)$ (see Figure $3 \mathrm{a}-\mathrm{d}$ ). Prior experiments on AB-BLG showed a kink in the $\mathrm{G}$ Raman frequency at $E_{\mathrm{F}} \sim 0.4 \mathrm{eV}$ that is associated 
with second sub-band filling. ${ }^{37}$ However, theoretical studies suggest an absence of sub-band between the VHSs of tBLG. ${ }^{11,12,50}$ Further studies are needed to understand the origin of these kinks in tBLG.

We now discuss the influence of asymmetric doping on the $2 \mathrm{D}$ and R Raman bands from the tBLG. These two bands are activated by intervalley DR process with phonon wavevector $\mathbf{q}$ $\neq 0 .{ }^{25,29,54}$ The $2 \mathrm{D}$ and $\mathrm{R}$ bands come from the same TO phonon branch but at different locations of the Brillouin zone (BZ). The $2 \mathrm{D}$ band originates from the scattering between the two adjacent Dirac cones ( $\mathrm{K}$ and $\mathrm{K}^{\prime}$ ) of a graphene layer with phonon wavevector $\mathbf{q}$ which is equal to the $K-K^{\prime}$ separation (same as $\Gamma-\mathrm{K}$ separation in the $\mathrm{BZ}$ ). The $\mathrm{R}$ band has a smaller phonon wavevector that equals the tBLG superlattice wavevector (see inset in Figure $4 \mathrm{~b}$ ). ${ }^{25,26}$
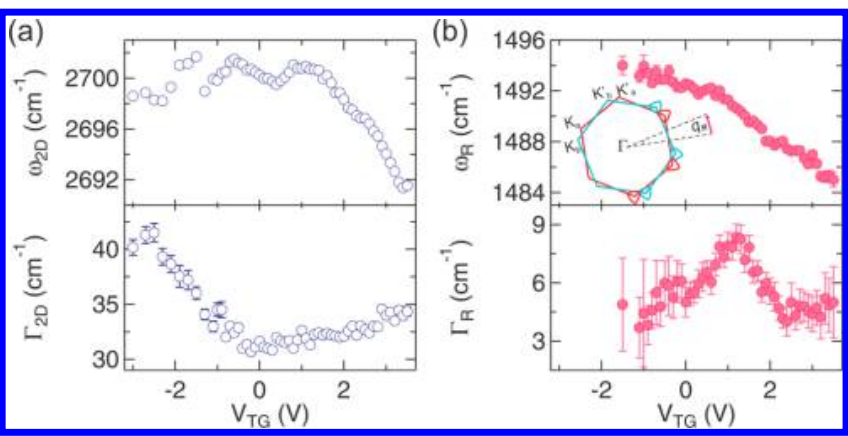

Figure 4. $(\mathrm{a}, \mathrm{b})$ Peak position $\left(\omega_{2 \mathrm{D}}, \omega_{\mathrm{R}}\right)$ and fwhm $\left(\Gamma_{2 \mathrm{D}}, \Gamma_{\mathrm{R}}\right)$ of $2 \mathrm{D}$ (left panel) and R (right panel) Raman bands as a function of $V_{\mathrm{TG}}$. The inset in (b) shows the first Brillouin zones of the top and bottom graphene layers rotated from each other by a twist angle of $\sim 13^{\circ}$. Wavevector of $\mathrm{R}$ phonon $\left(q_{\mathrm{R}}\right)$ is labeled.

Figure $4 \mathrm{a}$ shows the evolution of phonon frequency and fwhm of the $2 \mathrm{D}$ band as a function of $V_{\mathrm{TG}}$. All parameters are extracted from simple fits of the $2 \mathrm{D}$ band with single Lorentzian peaks. We obtain $\partial \omega_{2 \mathrm{D}} / \partial E_{\mathrm{F}} \sim-7 \mathrm{~cm}^{-1} \mathrm{eV}^{-1}$ for $\mathrm{n}$-doping and weak doping dependence for $\mathrm{p}$-doping in the tBLG. However, in the SLG (Supporting Information Figure S2) we obtain $\partial \omega_{2 \mathrm{D}} / \partial E_{\mathrm{F}} \sim 23 \mathrm{~cm}^{-1} \mathrm{eV}^{-1}$ for both $\mathrm{n}$ - and pdoping from our measurement, which is consistent with previous reports. ${ }^{36}$ The doping dependence of the fwhm of the $2 \mathrm{D}$ band in tBLG and SLG are different (Figure $4 \mathrm{a}$ and Supporting Information Figure S2b). For example, in high pdoping regime the fwhm of the tBLG increases by $\sim 10 \mathrm{~cm}^{-1}$ compared to its value at the CNP, but there is only a small variation (less than $3 \mathrm{~cm}^{-1}$ ) in the fwhm of the $2 \mathrm{D}$ band in the p-doped SLG (see Supporting Information Figure S2). The difference between the doping dependence of the $2 \mathrm{D}$ band of the $\mathrm{tBLG}$ and SLG may be linked to the difference in their band structures under an electric field. A change in the band structure of $\mathrm{tBLG}$ due to interlayer potential may have a large impact on the interaction of this second-order phonon with photons and electrons. Further studies are required to understand the mechanism causing the differences in the $2 \mathrm{D}$ band between tBLG and SLG.

A recent study of $\mathrm{tBLG}$ devices in which the twist angles are slightly smaller than the critical angle and electrodes are only in contact with one of the layers found that the 2D Raman band displays an asymmetric line shape that can be decomposed into two peaks with similar widths. ${ }^{55}$ The $2 \mathrm{D}$ splitting is attributed to different scattering pathways in DR process near the saddle points in the electronic band structure of $\mathrm{tBLG}^{55} \mathrm{We}$ did not observe such $2 \mathrm{D}$ band splitting in our tBLG devices. Precise reasons for this difference remain to be better understood but it may be related to several factors. First, the twist angle $\left(\sim 13^{\circ}\right)$ of our tBLG is slightly larger than the critical angle $\left(\sim 12.5^{\circ}\right)$ measured with $532 \mathrm{~nm}$ excitation laser energy. Second, both the top and bottom layers of our tBLG are in contact with the electrodes, which facilitate the alignment of $E_{\mathrm{F}}$ of the two layers when a $V_{\mathrm{TG}}$ is applied. In addition, the ion gel dielectrics $\left(\mathrm{PEO} / \mathrm{LiClO}_{4}\right)$ we used gives higher carrier densities $(\sim 3 \times$ $\left.10^{13} \mathrm{~cm}^{-2}\right)$ compared to those with $\mathrm{Si}$ backgate $\left(\sim 1 \times 10^{13}\right.$ $\left.\mathrm{cm}^{-2}\right) .35,36,55$ These differences may lead to distinct Raman features in our tBLG compared to those reported in recent literature. $^{55}$

Figure $4 \mathrm{~b}$ plots the frequency and fwhm of the $\mathrm{R}$ band as a function of $V_{\mathrm{TG}}$. The $\mathrm{R}$ band is too weak to be detected for heavily p-doped regime $\left(V_{\mathrm{TG}}<-1.5 \mathrm{~V}\right.$, Figure $\left.1 \mathrm{c}\right)$ and can only be fitted with a single Lorentzian function. The observed gate dependence of the $\mathrm{R}$ band frequency is similar to that of the $2 \mathrm{D}$ band. This may be linked to the fact that both bands are from the same TO phonon branch. We obtain $\partial \omega_{\mathrm{R}} / \partial E_{\mathrm{F}} \sim-9.2 \mathrm{~cm}^{-1}$ $\mathrm{eV}^{-1}$ for $\mathrm{n}$-doping which is slightly larger than that obtained from the $2 \mathrm{D}$ band, and $\partial \omega_{\mathrm{R}} / \partial E_{\mathrm{F}} \sim 3.8 \mathrm{~cm}^{-1} \mathrm{eV}^{-1}$ for $\mathrm{p}$-doping. The similar gate dependence of the $\mathrm{R}$ and $2 \mathrm{D}$ peak frequencies suggests that the phonon self-energy renormalization for the $\mathrm{R}$ band could share similar scattering mechanisms (e.g., a combination of electron-phonon and electron-electron interactions) as the $2 \mathrm{D}$ band. ${ }^{44}$ The gate-dependent frequency shift can be expressed using a phenomenological formula based on DFT calculation: ${ }^{56} \omega=a+b n_{\mathrm{TG}}+c n_{\mathrm{TG}}{ }^{2}+d n_{\mathrm{TG}}{ }^{3}+e\left|n_{\mathrm{TG}}\right|^{3 / 2}$, where $\omega$ is the phonon frequency, $n_{\mathrm{TG}}\left(\sim n_{\text {total }}\right.$ in low doping regime) is the effective carrier density (in unit of $10^{-13} \mathrm{~cm}^{-2}$ ) and $a, b, c, d$, and $e$ are coefficients. Fittings of gate dependence of the $\mathrm{R}$ and $2 \mathrm{D}$ frequencies to this phenomenological formula are shown in Supporting Information Figure S4, and the fitting parameters are summarized in Table $S 1$ in the Supporting Information. Prior studies have proposed that the twist angle of tBLG can be estimated from the frequencies of Raman $R$ and $\mathrm{R}^{\prime}$ bands. ${ }^{26,29,57}$ Our result on the $\mathrm{R}$ band suggests that doping level should be taken into account when determining the twist angle of $\mathrm{tBLG}$ via Raman measurements.

Although the gate dependence of the frequencies of the $2 \mathrm{D}$ and $\mathrm{R}$ bands are similar, the dependence of the fwhm of the two bands on the doping level is very different. The fwhm of the $\mathrm{R}$ band $\left(\Gamma_{\mathrm{R}}\right)$ reaches a maximum of $\sim 8 \mathrm{~cm}^{-1}$ at $V_{\mathrm{TG}} \sim 1 \mathrm{~V}$ $(\sim 0.5 \mathrm{~V}$ away from the $\mathrm{CNP})$ and then decreases for $V_{\mathrm{TG}}$ away from this value, including both highly $\mathrm{p}$ - and $\mathrm{n}$-doped regimes (Figure $4 \mathrm{~b}$ ). In contrast, the fwhm of the $2 \mathrm{D}$ band from tBLG shows a minimum at $\sim 0 \mathrm{~V}$ and increases rapidly in the p-doped regime. Further work is required to understand this difference.

In summary, novel features of the $G$ Raman band were observed in tBLG under gate tuning. In the presence of doping asymmetry (interlayer potential) in the two layers, a splitting of $\mathrm{G}$ Raman peak was observed. We determined the $E_{\mathrm{F}}$ and carrier concentration in each layer from the positions of the two $G$ peaks. We also observed a strong gate-dependent quenching of the $G$ peak intensities. It is interpreted by the suppression of interband direct transitions associated with the two low-energy saddle points (VHSs), which are oppositely shifted by interlayer potential, in the electronic structure of tBLG. An interlayer screening model was proposed to describe the observed phenomena, giving the effective interlayer capacitance of $\sim 4.6$ $\mu \mathrm{F} \mathrm{cm}{ }^{-2}$. The similarity of the gate dependence of the $2 \mathrm{D}$ and 
$\mathrm{R}$ frequencies suggests that the phonon self-renormalization of the $\mathrm{R}$ and $2 \mathrm{D}$ bands could share similar scattering mechanisms. Our findings demonstrated that doping asymmetry significantly alters the properties of tBLG. This gate modulation can therefore be used to control the physical properties of tBLG devices.

\section{ASSOCIATED CONTENT}

\section{S Supporting Information}

The material contains details about experimental procedures (graphene sample preparation, electrochemical top-gate field effect measurement and Raman spectroscopy), the method in determining the linear dependence of the $E_{\mathrm{F}}$ on the $G$ peak frequency in SLG, the doping dependence of the G and 2D bands in the SLG, the interlayer capacitance fitting from the interlayer potential in the bilayers, the fitting and corresponding parameters for the carrier density dependence of the $2 \mathrm{D}$ and $\mathrm{R}$ Raman peak frequencies in the tBLG, and the electrical field effect measurement of the SLG and tBLG devices. This material is available free of charge via the Internet at http://pubs.acs.org.

\section{AUTHOR INFORMATION}

\section{Corresponding Authors}

*E-mail: (T.-F.C.) tchung@purdue.edu.

*E-mail: (R.H.) rui.he@uni.edu.

\section{Notes}

The authors declare no competing financial interest.

\section{ACKNOWLEDGMENTS}

We acknowledge partial support of this work from NSF and DTRA. R.H. acknowledges support by the American Chemical Society Petroleum Research Fund (Grant 53401-UNI10), NSF Grant (DMR-1410496), and the UNI Faculty Summer Fellowship.

\section{REFERENCES}

(1) Geim, A. K.; Grigorieva, I. V. Van der Waals heterostructures. Nature 2013, 499, 419-425.

(2) Dean, C. R.; Young, A. F.; Lee, M. C.; Wang, L.; Sorgenfrei, S.; Watanabe, K.; Taniguchi, T.; Kim, P.; Shepard, K. L.; Hone, J. Boron nitride substrates for high-quality graphene electronics. Nat. Nanotechnol. 2010, 5, 722-726.

(3) Ponomarenko, L. A.; Geim, A. K.; Zhukov, A. A.; Jalil, R.; Morozov, S. V.; Novoselov, K. S.; Grigorieva, I. V.; Hill, E. H.; Cheianov, V. V.; Fal'ko, V. I.; Watanabe, K.; Taniguchi, T.; Gorbachev, R. V. Tunable metal-insulator transition in double-layer graphene heterostructures. Nat. Physics 2011, 7, 958-961.

(4) Yu, W. J.; Li, Z.; Zhou, H.; Chen, Y.; Wang, Y.; Huang, Y.; Duan, X. F. Vertically stacked multi-heterostructures of layered materials for logic transistors and complementary inverters. Nat. Mater. 2013, 12, 246-252.

(5) Georgiou, T.; Jalil, R.; Belle, B. D.; Britnell, L.; Gorbachev, R. V.; Morozov, S. V.; Kim, Y.-J.; Gholinia, Al.; Haigh, S. J.; Makarovsky, O.; Eaves, L.; Ponomarenko, L. A.; Geim, A. K.; Novoselov, K. S.; Mishchenko, A. Vertical field-effect transistor based on graphene-WS heterostructures for flexible and transparent electronics. Nat. Nanotechnol 2013, 8, 100-103.

(6) Britnell, L.; Ribeiro, R. M.; Eckmann, A.; Jalil, R.; Belle, B. D.; Mishchenko, A.; Kim, Y.-J.; Gorbachev, R. V.; Georgiou, T.; Morozov, S. V.; Grigorenko, A. N.; Geim, A. K.; Casiraghi, C.; Castro Neto, A. H.; Novoselov, K. S. Strong Light-Matter Interactions in Heterostructures of Atomically Thin Films. Science 2013, 340, 1311-1314.

(7) Fang, H.; Battaglia, C.; Carraro, C.; Nemsak, S.; Ozdol, B.; Kang, J. S.; Bechtel, H. A.; Desai, S. B.; Kronast, F.; Unal, A. A.; Conti, G.; Conlon, C.; Palsson, G. K.; Martin, M. C.; Minor, A. M.; Fadley, C. S.;
Yablonovitch, E.; Maboudian, R.; Javey, A. Strong interlayer coupling in van der Waals heterostructures built from single-layer chalcogenides. Proc. Natl. Acad. Sci. U.S.A. 2014, 111, 6198-6202.

(8) Britnell, L.; Gorbachev, R. V.; Jalil, R.; Belle, B. D.; Schedin, F.; Mishchenko, A.; Georgiou, T.; Katsnelson, M. I.; Evaes, L.; Morozov, S. V.; Peres, N. M. R.; Leist, J.; Geim, A. K.; Novoselov, K. S.; Ponomarenko, L. A. Field-Effect Tunneling Transistor Based on Vertical Graphene Heterostructures. Science 2012, 335, 947-950.

(9) Sanchez-Yamagishi, J. D.; Taychatanapat, T.; Watanabe, K.; Taniguchi, T.; Yacoby, A.; Jarillo-Herrero, P. Quantum Hall Effect, Screening, and Layer-Polarized Insulating States in Twisted Bilayer Graphene. Phys. Rev. Lett. 2012, 108, 076601.

(10) Li, G.; Luican, A.; dos Santos, J.; Neto, A.; Reina, A.; Kong, J.; Andrei, E. Observation of Van Hove singularities in twisted graphene layers. Nat. Phys. 2010, 6, 109-113.

(11) Lopes dos Santos, J. M. B; Peres, N. M. R.; Castro, A. H. Graphene Bilayer with a Twist: Electronic Structure. Phys. Rev. Lett. 2007, 99, 256802.

(12) Shallcross, S.; Sharma, S.; Kandelaki, E.; Pankratov, O. Electronic structure of turbostratic graphene. Phys. Rev. B 2010, 81, 165105.

(13) Ohta, T.; Robinson, J. T.; Feibelman, P. J.; Bostwick, A.; Rotenberg, E.; Beechem, T. E. Evidence for Interlayer Coupling and Moiré Peridoic Potentials in Twisted Bilayer Graphene. Phys. Rev. Lett. 2012, 109, 186807.

(14) Chu, Z. D.; He, W. Y.; He, L. Coexistence of van Hove singularities and superlattice Dirac points in a slightly twisted graphene bilayer. Phys. Rev. B 2013, 87, 155419.

(15) Moon, P.; Koshino, M. Optical absorption in twisted bilayer graphene. Phys. Rev.B 2013, 87, 205404.

(16) Luican, A.; Li, G.; Reina, A.; Kong, J.; Nair, R.; Novoselov, K.; Geim, A.; Andrei, E. Single-Layer Behavior and Its Breakdown in Twisted Graphene Layers. Phys. Rev. Lett. 2011, 106, 126802.

(17) Brihuega, I.; Mallet, P.; Gonzalez-Herrero, H.; de laissardiere, G.; ugeda, M.; Magaud, L.; Gomez-Rodriguez, J.; Yndurain, F.; Veuillen, J. Unraveling the Intrinsic and Robust Nature of van Hove Singularities in Twisted Bialyer Graphene by Scanning Tunneling Microscopy and Theoretical Analysis. Phys. Rev. Lett. 2012, 109, 196802.

(18) Meng, L.; Yan, W.; Yin, L. J.; Chu, Z. D.; Zhang, Y. F.; Feng, L.; Dou, R. F.; Nie, J. C. Tuning the Interlayer Coupling of the Twisted Bilayer Graphene by Molecular Adsorption. J. Phys. Chem. C 2014, $118,6462-6466$.

(19) Ni, Z.; Liu, L.; Wang, Y.; Zheng, Z.; Li, L.; Yu, T.; Shen, Z. Gband Raman double resonance in twisted bialyer grpahene: Evidence of band splitting and folding. Phys. Rev. B 2009, 80, 125404.

(20) Wang, Y. Y.; Ni, Z. H.; Liu, L.; Liu, Y. H.; Cong, C. X.; Yu, T.; Wang, X. J.; Shen, D. Z.; Shen, Z. X. Stacking-Dependent Optical Conductivity of Bilayer Graphene. ACS Nano 2010, 4, 4074-4080.

(21) Kim, K.; Coh, S.; Tan, L.; Regan, W.; Yuk, J.; Chatterjee, E.; Crommie, M.; Cohen, M.; Louie, S.; Zettl, A. Raman Spectroscopy Study of Rotated Double-Layer Graphene: Misorientation-Angle Dependence of Electronic Structure. Phys. Rev. Lett. 2012, 108, 246103.

(22) Havener, R. W.; Zhuang, H. L.; Brown, L.; Hennig, R. G.; Park, J. Angle-Resolved Raman Imaging of Interlayer Rotations and Interactions in Twisted Bilayer Graphene. Nano Lett. 2012, 12, $3162-3167$

(23) Robinson, J. T.; Schmucker, S. W.; Diaconescu, C. B.; Long, J. P.; Culbertson, J. C.; Ohta, T.; Friedman, A. L.; Beechem, T. E. Electronic Hybridization of Large-Area Stacked Graphene Films. ACS Nano 2012, 7, 637-644.

(24) Havener, R. W.; Liang, Y. F.; Brown, L.; Yang, L.; Park, J. Van Hove Singularities and Excitonic Effects in the Optical Conductivity of Twisted Bilayer Graphene. Nano Lett. 2014, 14, 3353-3357.

(25) Carozo, V.; Almeida, C. M.; Ferreira, E. H. M.; Cançado, L. G.; Achete, C. A.; Jorio, A. Raman Signature of Graphene Superlattices. Nano Lett. 2011, 11, 4527-4534. 
(26) Jorio, A.; Cançado, L. G. Raman spectroscopy of twisted bilayer graphene. Solid State Commun. 2013, 175-176, 3-12.

(27) He, R.; Chung, T. F.; Delaney, C.; Keiser, C.; Jauregui, L. A.; Shand, P. M.; Chancey, C. C.; Wang, Y.; Bao, J.; Chen, Y. P. Observation of Low Energy Raman Modes in Twisted Bilayer Graphene. Nano Lett. 2013, 13, 3594-3601.

(28) Gupta, A. K.; Tang, Y.; Crespi, V. H.; Eklund, P. C. Nondispersive Raman D band activated by well-ordered interlayer interactions in rotationally stacked bilayer graphene. Phys. Rev. B 2010, 82,241406

(29) Carozo, V.; Almeida, C. M.; Fragneaud, B.; Bedê, P.; Moutinho, J.; Riberiro-Soares, M. V. O.; Andrade, N.; Souza Filho, A. G.; Matos, M. J. S.; Wang, B.; Terrones, M.; Capaz, R. B.; Jorio, A.; Achete, C. A.; Cançado, L. G. Resonance effects on the Raman spectra of graphene superlattices. Phys. Rev. B 2013, 88, 085401.

(30) Campos-Delgado, J.; Cançado, L. G.; Achete, C. A.; Jorio, A.; Raskin, J.-P. Raman scattering study of the phonon dispersion in twisted bilayer graphene. Nano Res. 2013, 6, 269-274.

(31) Righi, A.; Costa, S. D.; Chacham, H.; Fantini, C.; Venezuela, P.; Magnuson, C.; Colombo, L.; Bacsa, W. S.; Ruoff, R. S.; Pimenta, M. A. Graphene Moiré patterns observed by umklapp double-resonance Raman scattering. Phys. Rev. B 2011, 84, 241409.

(32) Yan, J.; Villarson, T.; Henriksen, E. A.; Kim, P.; Pinczuk, A. Optical phonon mixing in bilayer graphene with a broken inversion symmetry. Phys. Rev. B 2009, 80, 241417.

(33) Malard, L. M.; Elias, D. C.; Alves, E. S.; Pimenta, M. A. Observation of Distinct Electron-Phonon Couplings in Gated Bilayer Graphene. Phys. Rev. Lett. 2008, 101, 257401.

(34) Moon, P.; Son, Y. W.; Koshino, M. Optical absorption of twisted bilayer graphene with interlayer potential asymmetry. Phys. Rev. B 2014, 90, 155427.

(35) Araujo, P. T.; Frank, O.; Mafra, D. L.; Fang, W.; Kong, J.; Dresselhaus, M. S.; Kalbac, M. Mass-related inversion symmetry breaking and phonon self-energy renormalization in isotopically labeled AB-stacked bilayer graphene. Sci. Rep. 2013, 3, 2061.

(36) Das, A.; Pisana, S.; Chakraborty, B.; Piscanec, S.; Saha, S. K.; Waghmare, U. V.; Novoselov, K. S.; Krishnamurthy, H. R.; Geim, A. K.; Ferrari, A. C.; Sood, A. K. Monitoring dopants by Raman scattering in an electrochemically top-gated graphene transistor. Nat. Nanotechnol. 2008, 3, 210-215.

(37) Das, A.; Chakraborty, B.; Piscanec, S.; Pisana, S.; Sood, A. K.; Ferrari, A. C. Phonon renormalization in doped bilayer graphene. Phys. Rev. B 2009, 79, 155417.

(38) Yan, J.; Zhang, Y.; Kim, P.; Pinczuk, A. Electric Field Effect Tuning of Electron-Phonon Coupling in Graphene. Phys. Rev. Lett. 2007, 98, 166802 .

(39) Mohiuddin, T. M.G.; Lombardo, A.; Nair, R. R.; Bonetti, A.; Savini, G.; Jalil, R.; Bonini, N.; Basko, D. M.; Galiotis, C.; Marzari, N.; Novoselov, K. S.; Geim, A. K.; Ferrari, A. C. Uniaxial strain in graphene by Raman spectroscopy: G peak splitting, Grüneisen parameters, and sample orientation. Phys. Rev. B 2009, 79, 205433.

(40) Huang, M.; Yan, H.; Chen, C.; Song, D.; Heinz, T. F.; Hone, J. Phonon softening and crystallographic orientation of strained graphene studied by Raman spectroscopy. Proc. Natl. Acad. Sci. U.S.A. 2009, 106, 7304-7308.

(41) He, R.; Zhao, L. Y.; Petrone, N.; Kim, K. S.; Roth, M.; Hone, J.; Kim, P.; Pasupathy, A.; Pinczuk, A. Large Physisorption Strain in Chemical Vapor Deposition of Graphene on Copper Substrates. Nano Lett. 2012, 12, 2408-2413.

(42) Kalbac, M.; Farhat, H.; Kong, J.; Janda, P.; Kavan, L.; Dresselhaus, M. S. Raman Spectroscopy and in Situ Raman Spectroelectrochemistry of Bilayer ${ }^{12} \mathrm{C} /{ }^{13} \mathrm{C}$ Graphene. Nano Lett. 2011, 11, 1957-1963.

(43) Basko, D. M. Theory of resonant multiphonon Raman scattering in graphene. Phys. Rev. B 2008, 78, 125418.

(44) Basko, D. M.; Piscanec, S.; Ferrari, A. C. Electron-electron interactions and doping dependence of the two-phonon Raman intensity in graphene. Phys. Rev. B 2009, 80, 165413.
(45) Childres, I.; Jauregui, L. A.; Chen, Y. P. Raman spectra and electron-phonon coupling in disordered graphene with gate-tunable doping. J. Appl. Phys. 2014, 116, 233101.

(46) Pisana, S.; Lazzeri, M.; Casiraghi, C.; Novoselov, K. S.; Geim, A. K.; Ferrari, A. C.; Mauri, F. Breakdown of the adiabatic BornOppenheimer approximation in graphene. Nat. Mater. 2007, 6, 198201.

(47) Chen, C.-F.; Park, C.-H.; Boudouris, B. W.; Horng, J.; Geng, B.; Girit, C.; Zettl, A.; Crommie, M. F.; Segalman, R. A.; Louie, S. G.; Wang, F. Controlling inelastic light scattering quantum pathways in graphene. Nature 2011, 471, 617.

(48) Gava, P.; Lazzeri, M.; Saitta, A. M.; Mauri, F. Ab initio study of gap opening and screening effects in gated bilayer graphene. Phys. Rev. B 2009, 79, 165431.

(49) Kuroda, M. A.; Tersoff, J.; Martyna, G. J. Nonlinear Screening in Multilayer Graphene Systems. Phys. Rev. Lett. 2011, 106, 116804.

(50) San-Jose, P.; Prada, E. Helical networks in twisted bilayer graphene under interlayer bias. Phys. Rev. B 2013, 88, 121408.

(51) Yang, L. Excitonic Effects on Optical Absorption Spectra of Doped Graphene. Nano Lett. 2011, 11, 3844-3847.

(52) Santos, E. J. G.; Kaxiras, E. Electric-Field Dependence of the Effective Dielectric Constant in Graphene. Nano Lett. 2013, 13, 898902.

(53) Ryu, S.; Liu, L.; Berciaud, S.; Yu, Y.-J.; Liu, H.; Kim, P.; Flynn, G. W.; Brus, L. E. Atmosphereic Oxygen Binding and Hole Doping in Deformed Graphene on a $\mathrm{SiO}_{2}$ Substrate. Nano Lett. 2010, 10, 49444951.

(54) Araujo, P. T.; Mafra, D. L.; Sato, K.; Saito, R.; Kong, J.; Dresselhaus, M. S. Phonon Self-Energy Corrections to Nonzero WaveVector Phonon Modes in Single-Layer Graphene. Phys. Rev. Lett. 2012, 109, 046801.

(55) Yeh, C.-H.; Lin, Y.-C.; Chen, Y. -C; Lu, C.-C.; Liu, Z.; Suenaga, K.; Chiu, P.-W. Gating Electron-Hole Asymmetry in Twisted Bilayer Graphene. ACS Nano 2014, 8, 6962.

(56) Lazzeri, M.; Mauri, F. Nonadiabatic Kohn Anomaly in a Doped Graphene Monolayer. Phys. Rev. Lett. 2006, 97, 266407.

(57) Coh, S.; Tan, L. Z.; Louie, S. G.; Cohen, M. L. Theroy of the Raman spectrum of rotated double-layer graphene. Phys. Rev. B 2013, 88,165431 . 